Revista de Investigación en Logopedia ISSN: $1131-8635$

http://dx.doi.org/10.5209/RLOG.58249

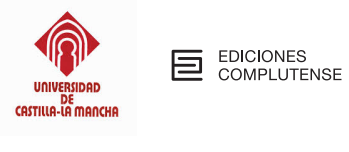

\title{
Cuestionario de adherencia para las adaptaciones dietéticas en disfagia
}

\author{
Núria Oriol-Peregrina ${ }^{1}$
}

Recibido: 5 de diciembre de 17 / Revisado: 27 de febrero de 2018 / Aceptado: 21 de mayo de 2018.

Resumen. Las adaptaciones de la dieta son estrategias adaptativas de compensación que se utilizan para optimizar la seguridad y la eficacia deglutoria en personas que presentan disfagia. El objetivo del artículo es proponer un instrumento para mejorar el continuum asistencial en la práctica clínica en personas con alteraciones de la deglución que requieren adaptaciones dietéticas, dado que en la literatura no existe ningún instrumento de estas características. En el diseño se combinan distintas metodologías, en las que los enfoques cualitativo y cuantitativo de investigación se mezclan en un único procedimiento de investigación mixta. Se realiza el procedimiento del Método Delphi para la búsqueda de consenso en la búsqueda de evidencias de validación basadas en el contenido de los ítems. Un total de 13 expertos a través de dos rondas llegan a un CVI-I igual a 1 en un 100\% de las cuestiones presentadas sobre el contenido del Cuestionario de Adherencia. El instrumento propuesto inicialmente se modifica y mejora gracias a las opiniones de los expertos. El resultado final es un Cuestionario de Adherencia para utilizar en la práctica clínica con personas que padecen disfagia y necesitan adaptaciones en la dieta, consensuado por un grupo de expertos.

Palabras clave: trastornos de deglución; dietoterapia; adaptaciones dietéticas; líquidos espesados; adherencia al tratamiento, cuestionario.

\section{[en] Adherence questionnaire for dietary adaptations in dysphagia}

\begin{abstract}
Dietary adaptations are adaptive compensation strategies that are used to optimize safety and swallowing efficacy on people who have dysphagia. The aim of the article is to propose an instrument to improve the continuum of care in clinical practice in people with swallowing disorders that require dietary adaptations, given that in the literature there is no instrument of these characteristics. In the design, different methodologies are combined, in which the qualitative and quantitative research approaches are mixed in a single mixed research procedure. The procedure of the Delphi Method is performed to search for consensus in the search for validation evidences based on the content of the items. A total of 13 experts through two rounds arrive at a CVI-I equal to $1 \mathrm{in} 100 \%$ of the questions presented on the content of the Adherence Questionnaire. The originally proposed instrument is modified and improved thanks to the opinions of the experts. The final result is an Adherence Questionnaire to be used in clinical practice with people suffering from dysphagia and who need adaptations in the diet, agreed by a group of experts.
\end{abstract}

Key words: deglutition disorders; diet therapy; texture-modification, thickener-fluid; patient compliance; questionnaire.

Cómo citar: Oriol-Peregrina, N. (2018). Adherence questionnaire for dietary adaptations in dysphagia. Revista de Investigación en Logopedia, 8(2), 91-114.

Universidad de Manresa, UVic-UCC. España.

noriol@umanresa.cat 


\section{Introducción}

La disfagia es la alteración en la deglución de alimentos sólidos, líquidos y / o saliva (Bleeckx, 2004, p. 80) y se define como una alteración del sistema biomecánico del acto de tragar por incoordinación del proceso, obstrucción del paso, debilidad o rigidez muscular (Smith Hammond y Goldstein, 2006) con diferentes niveles de gravedad que pueden abarcar desde la situación más grave que deriva en la imposibilidad de alimentación por vía oral, hasta otros de menor riesgo en pacientes que pueden seguir alimentándose por vía oral siempre que se tomen determinadas medidas.

La deshidratación, la desnutrición, y las broncoaspiraciones o neumonías broncoaspirativas que pueden llegar a producir la muerte son las consecuencias asociadas a la disfagia (Almirall, Cabré, y Clavé, 2007; Clavé y Cichero, 2012; Clavé y García Peris, 2011; Clavé, Verdaguer, y Arreola, 2005; Ickenstein, 2011). Al ser éstas graves conviene intervenir para favorecer una mejora en la salud.

El abanico de tratamientos y técnicas terapéuticas para mejorar y / o superar la disfagia es amplio, contempla desde simples recomendaciones para ayudar en la seguridad deglutoria, hasta intervenciones quirúrgicas en casos más graves. Las adaptaciones de la dieta son estrategias adaptativas de compensación que se utilizan para optimizar la seguridad y la eficacia deglutoria sin modificar el mecanismo fisiológico del proceso deglutorio. Consisten en modificar las texturas de los alimentos sólidos y la viscosidad de los líquidos. Pueden implementarse de forma rápida para minimizar o anular las penetraciones y aspiraciones cuando el paciente se alimenta por vía oral, de la misma forma que pueden retirarse cuando dejan de ser necesarias. Otra ventaja es que se pueden instaurar cuando el paciente tiene comprometidas las funciones superiores y no puede seguir otro tipo de intervención (Ickenstein, 2011). Pueden utilizarse de forma aislada o combinadas con otras técnicas de intervención. De este modo, son la principal herramienta para favorecer la seguridad y la eficacia deglutoria en aquellos pacientes que no pueden colaborar, y se pueden utilizar conjuntamente con las estrategias compensatorias y las técnicas de rehabilitación en aquellos pacientes en los que sí existe cierto grado de colaboración.

Una de las dificultades en la instauración de las adaptaciones dietéticas es la generalización y el uso mantenido en el tiempo. El cuestionario de adherencia permite constatar a posteriori el seguimiento o no de las indicaciones pactadas y los motivos que pueden dificultar su instauración. Con esta información, el logopeda puede reorientar las indicaciones para favorecer el tratamiento más adecuado para el paciente.

Para conseguir la seguridad deglutoria es importante introducir el modo de tomar la medicación por el riesgo que supone tragar pastillas, cápsulas o ampollas. Existen procedimientos para decidir la mejor forma farmacéutica y método de administración de medicamentos (Aguilar Salmeron R., s. f.; Hernández Martín et al., 2013; Wright et al., 2015).

Así mismo, es necesario apuntar la forma en que se realiza la higiene oral ya que las personas con disfagia a líquidos pueden atragantarse cuando se aclaran con el agua o el colutorio al lavarse los dientes (Scottish Intercollegiate Guidelines Network, 2004), además de que una mala higiene oral puede aumentar la carga bacteriana del material que se aspira (Ortega, Cabré, y Clavé, 2014).

Como en la mayoría de intervenciones terapéuticas en las que el fin último es no sólo informar, sino educar y concienciar sobre una determinada cuestión, la simple 
utilización de instrumentos para favorecer este objetivo es insuficiente. La información que se comparte, el espacio que se ofrece para que pacientes y familias puedan expresar sus preferencias y valores, el vínculo que se teje entre el logopeda y el paciente-familia acaban siendo el pilar fundamental en el éxito del seguimiento de las medidas indicadas. A través del diálogo, con la simbología del lenguaje que permite expresar percepciones, sentimientos e ideas, la comunicación puede hacerse más efectiva ayudando a interpretar los significados de las acciones.

\section{Antecedentes}

Existen múltiples instrumentos para valorar la adherencia a los tratamientos médicos farmacológicos como por ejemplo el "Test de cumplimiento autocomunicado o Test de Haynes-Sackett" (Haynes et al., 1980), el "Test de Morisky- Grenn" (Morisky, Green, y Levine, 1986) entre otros.

También existen varias herramientas para valorar el seguimiento a determinadas dietas, las cuales se centran en tres grandes grupos. Un primer grupo destinado a recoger el seguimiento de la dieta mediterránea, como por ejemplo la "Predimed" (Centro de Investigación Biomédica en Red, 2015); un segundo en el que se engloban las que están en el entorno de patologías concretas como las hepatopatías o factores de riesgo como el colesterol o la hipertensión, siendo una muestra el "Dietary Sodium Restriction Questionnaire" (Bentley, Lennie, Biddle, Chung, y Moser, 2009); y un tercer grupo de caràcter mas general (Russell, Flood, Sadeghpour, Gopinath, y Mitchell, 2017).

Actualmente no se dispone de ningún test de adherencia terapéutica en pacientes que tienen que adaptar su dieta debido a la disfagia. La búsqueda de artículos en la base de datos pubmed el día 25 de agosto de 2016 desde el año 2010 con las palabras clave "test", "questionnaire", "adherence", "compliance" y "dysphagia" no ofrece resultados en este tema. Por este motivo se presenta una herramienta que permita recoger el grado de adherencia, entendiendo que ésta forma parte del continuum asistencial.

\section{Método}

\section{Diseño del estudio}

Se parte del paradigma investigación-acción, orientado a la práctica, en el que se "prioriza la acción, la investigación y la práctica cotidiana sobre la teoría" (Tójar Hurtado, 2006). Son exponentes Stenhouse que cuestiona los fenómenos sociales desde lo habitual y los lleva al ámbito filosófico (Stenhouse, 1996), o Elliot que busca la comprensión de las situaciones sociales y educativas conflictivas para modificarlas a través de las acciones (Elliot, 2000). La elaboración del cuestionario de adherencia se caracteriza específicamente por hacer algo para mejorar la práctica.

En este estudio se combinan distintas metodologías, en las que los enfoques cualitativo y cuantitativo de investigación se mezclan en un único procedimiento de investigación mixta. Se necesitan dos rondas de consulta aplicando el procedimiento 
del Método Delphi para la búsqueda de consenso en la búsqueda de evidencias de validación basadas en el contenido de los ítems.

\section{Objetivo general}

Proponer un instrumento para mejorar el continuum asistencial en la práctica clínica en personas con disfagia que requieren adaptaciones dietéticas.

\section{Objetivos específicos}

1) Diseñar un cuestionario para recoger el grado de adherencia terapéutica a las recomendaciones dietéticas proporcionadas a los pacientes con disfagia.

2) Aportar evidencias de validez basadas en el contenido del Cuestionario de adherencia.

\section{Población a la que va dirigido}

Personas que presentan disfagia y a las que se les ha recomendado una adaptación de la dieta como uno de los posibles tipos de intervención.

\section{Procedimiento}

\section{1) Desarrollo del contenido del cuestionario para recoger el grado de adherencia a las adaptaciones dietéticas.}

La definición conceptual del constructo del Cuestionario de Adherencia (CA) y la preparación inicial de las preguntas que lo componen derivan de la revisión de diferentes teorías de educación para la salud, y de la experiencia clínica de la investigadora. En cuanto al examen de las teorías de la salud se consideran el Modelo transteórico del cambio que aporta las diferentes etapas en las que discurre el paciente respecto la disposición al cambio y en función de las cuales se plantean unas intervenciones específicas (precontemplación, contemplación, preparación para el cambio, acción, mantenimiento, finalización, recaída, siendo potencialmente incumplidores los que están en las tres primeras etapas) (Prochaska, 1979); la Teoría de la acción razonada que recoge la importancia de la intención determinada por unas creencias (Fishbein y Ajzen, 1975); el Modelo de creencias en salud que contribuye a sopesar los beneficios y las dificultades percibidas en la adherencia a los tratamientos (Janz y Becker, 1984; Rosenstock, 1974); y la Teoría social Cognitiva de Bandura por el concepto de autoeficacia y de apoyo social (Bandura, 1987).

El cuestionario de adherencia se define en cinco secciones.

En un primer apartado se recogen datos personales, las fechas (de cuando se hicieron las orientaciones dietéticas y la del actual cuestionario) y demás información para contextualizar el desarrollo de la alimentación (tipo de adaptación dietética, cómo se administra la medicación, tipo de higiene oral recomendada. quien escoge los alimentos, quien cocina, lugar habitual de las comidas). Por último, se recoge la etapa de cambio en la que el paciente-familia se encuentra, pues permitirá adaptar el discurso al momento que están viviendo en relación con los cambios solicitados en la dieta. 
El segundo apartado es propiamente el cuestionario de adherencia. Se pide sobre acciones y en pasado, pues el objetivo es recoger el seguimiento a posteriori de haber iniciado en el domicilio o en el lugar habitual de residencia la dieta introducida. Este tiempo puede ser variable, y en función de cada caso, al cabo de quince días, al cabo de un mes, etc.

Se definen las conductas de riesgo, que son: comer los alimentos de los grupos de alimentos difíciles, no seguir las indicaciones de viscosidad de los líquidos, no tomar la medicación como se recomienda, no lavarse los dientes después de cada comida.

Se comienza con una pregunta abierta, no inducida por el profesional, para obtener información directa del paciente-familia. Al mismo tiempo se pide que se concreten las comidas hechas en un día para tener un ejemplo de cómo se está alimentando.

Se sigue con una pregunta de respuesta cerrada para saber si ha seguido las recomendaciones indicadas. De este modo se recoge el posicionamiento en relación a las adaptaciones. En caso de que la respuesta sea "no", se pide el motivo (no me gusta, me he cansado, no pienso que sea necesario...).

A continuación, se cuestiona sobre la ingesta de alimentos considerados de riesgo. Son 9 preguntas, una por cada grupo de alimentos de riesgo. Se dan cuatro opciones de respuesta según la frecuencia, cuantificando las opciones: nunca, alguna vez (entre 1-2 veces a la semana), a menudo (entre 3-7 veces a la semana), siempre (cada vez que ha querido). La respuesta esperada sería "nunca", en el caso de haber seguido las recomendaciones pactadas. En la tabla 1 se presentan los grupos de alimentos de riesgo que orientan la dieta de la persona con disfagia y fundamenta las 9 preguntas.

Tabla 1 Grupos de alimentos agrupados por texturas

\begin{tabular}{l|l}
\hline \multicolumn{1}{c|}{ TIPO DE ALIMENTO } & \multicolumn{1}{c}{ EJEMPLOS } \\
\hline Fibroso & Piña, apio, hojas duras de lechuga \\
\hline Alimentos con partes duras o que pinchan & $\begin{array}{l}\text { Carne con hueso, tendones, cartílagos, frutos secos, pes- } \\
\text { cado con espinas }\end{array}$ \\
\hline Pieles y tegumentos de fruta, legumbres & Uvas, garbanzos, judías \\
\hline Alimentos de diferentes consistencias & $\begin{array}{l}\text { Leche con muesli o cereales de desayuno; pan de cereales, } \\
\text { sopa con pasta, verduras o trozos de carne, cocido }\end{array}$ \\
\hline Alimentos que desprenden agua o que se deshacen & Naranja, mandarina, sandía, melón, helado \\
\hline Alimentos crujientes o que se desmenuzan & $\begin{array}{l}\text { Patatas chips, corteza de pan, pan tostado o biscotes, ga- } \\
\text { lletas maría }\end{array}$ \\
\hline Alimentos secos & Pechuga de pollo a la plancha, torta \\
\hline Alimentos pegajosos & $\begin{array}{l}\text { Caramelos masticables o tofes, turrón de Jijona, miel, } \\
\text { puré de patatas espeso }\end{array}$ \\
\hline \hline
\end{tabular}

Nota: Tomada de (Clavé y García Peris, 2011, p. 117; Prados y Piera, 2014; Scottish Intercollegiate Guidelines Network, 2004, p. 31). Adaptada. 
A continuación, consta una pregunta referente a la frecuencia en la ingesta de líquidos. Las opciones de respuesta siguen siendo: nunca, alguna vez (entre 1-2 veces a la semana), a menudo (entre 3-7 veces a la semana), siempre. Aunque la escala de respuesta en esta pregunta tenga el sentido contrario, "siempre" se refiere a la respuesta esperada si han hecho la viscosidad recomendada, se considera que la pregunta es de más fácil comprensión y respuesta si se hace en positivo.

Se recogen las conductas en relación a dos aspectos relacionados como son la administración de la medicación y la higiene bucal. En ambos casos se proponen opciones de respuesta para facilitar la recogida de la información.

El tercer apartado cuestiona a través de una pregunta abierta la vivencia del paciente, para entender la etapa de cambio en la que está instalado. La respuesta permitirá ayudar a reconducir el discurso para favorecer aquello que sea mejor para el paciente, por ejemplo si hay recaídas.

El cuarto apartado permite obtener información a través de preguntas de futuro de respuesta sí/no y razonamiento posterior la intención del mantenimiento de las recomendaciones, concepto clave en el mantenimiento de las conductas esperadas a adherir de la teoría de la acción razonada, así como de los beneficios-dificultades percibidas. Esta última ofrece información sobre la autoeficacia, concepto clave en el mantenimiento de las conductas esperadas a adherir según la teoría de Bandura.

En quinto y último lugar se valora el grado de adherencia de forma cualitativa. El objetivo es definir si se da adherencia o no, y en caso de que sí se dé detectar si aparece siempre (buena), si se da a veces (parcial), si es esporádica o si a partir de un momento determinado deja de mantenerse (secuencial) (Vilaplana, González, y Ordoñana, 2012). De esta forma se puede obtener información para reorientar el discurso y la comunicación con la unidad paciente-familia.

Las preguntas van dirigidas al paciente si cognitivamente está bien, y también al familiar, ya que puede existir falta de concordancia entre sus respuestas. Las respuestas se recogen en el cuestionario de la siguiente forma: rodeando la respuesta adecuada cuando haya opciones, redactando en caso de preguntas abiertas y marcando con una cruz las preguntas de la 3 a la 11. Las respuestas del paciente se recogen en color rojo y las respuestas del familiar en color verde. Estas indicaciones junto con otra información para utilizar de forma óptima el CA se ofrecen de forma complementaria en una Hoja de instrucciones adjunta. El Cuestionario de Adherencia y la Hoja de instrucciones se encuentran en el apéndice.

\section{2) Pruebas de validez basadas en el contenido del cuestionario}

Para las pruebas de validez basadas en el contenido del CA se tienen en cuenta las distintas recomendaciones publicadas (American Educational Research Association, American Psychological Association, y National Council on Measurement in Education, 2014; Pernambuco, Espelt, Magalhães Junior, y Lima, 2017). El contenido se refiere a los temas, redacción, formato, tareas o preguntas en la prueba, así como las instrucciones para los procedimientos necesarios para administrarlo y marcarlo. Las estrategias utilizadas para evaluar el CA han sido la revisión de la literatura, la experiencia empírica de la investigadora principal y entrevistas con informantes clave empleando la técnica Delphi. También deben considerarse los aspectos sintácticos y semánticos en la elaboración de los ítems, así como la pertinencia, coherencia y alcance de éstos. 
El procedimiento para obtener las pruebas de validez basadas en el contenido del cuestionario contiene dos pasos clave: la elaboración del cuestionario para evaluar el CA y la metodología de consenso con panel de expertos. Los resultados se analizan según los elementos que evalúan la calidad de contenido de un instrumento: definición de dominio, representación de dominio y relevancia del dominio (Alexandre y Coluci, 2011; Sireci, 1998).

El dominio "proporciona los detalles sobre lo que mide la prueba y transforma la construcción teórica en un dominio de contenido más concreto" (Sireci, 1998). En el caso del CA se establece el dominio en la adherencia de las personas con disfagia a las adaptaciones dietéticas. El dominio de representación "se refiere al grado en que la prueba representa y mide el dominio tal cómo se define en la especificación de la prueba" (Sireci, 1998). Por lo tanto, debe observarse si el CA representa realmente el dominio acabado de definir. Finalmente la relevancia del dominio "aborda hasta qué punto cada elemento de la prueba es relevante para el dominio específico" (Sireci, 1998). En este sentido, se analizan los diferentes elementos para examinar su relevancia para el dominio definido.

\section{Elaboración del cuestionario para evaluar el Cuestionario de Adherencia}

Esta segunda fase corresponde a la elaboración del cuestionario para valorar el CA, que contiene un total de 20 ítems o afirmaciones (tabla 2). En primer lugar se recoge el número de registro que corresponde al número de identificación de cada experto. Las afirmaciones contienen información sobre el ajuste de la información solicitada, sobre los aspectos formales de las preguntas, si la temporalidad expresada en recoger el seguimiento es adecuada, acerca de la capacidad y voluntad de mantener la conducta esperada por parte del paciente y si las categorías para recoger los grados de adherencia son apropiadas. En último lugar se ofrece una última pregunta abierta para complementar los comentarios que no hayan sido contemplados en las preguntas anteriores.

Todos los elementos se presentan en forma de afirmaciones que son las que deben ser evaluadas de forma categorizada en una escala Likert (Pernambuco et al., 2017). Las posibles respuestas son: 1 = "Muy en desacuerdo", 2 = "En desacuerdo", 3 = "Indiferente", 4 = "De acuerdo " y 5 = "Muy de acuerdo". En todos los ítems se destina un espacio para aportar apreciaciones explicativas y así recoger de forma cualitativa la opinión de los expertos, especialmente para cuando expresan un desacuerdo. 
Tabla 2. Cuestionario para evaluar el Cuestionario de Adherencia. Número de expertos que responden a cada ítem en las dos olas según la escala Likert

\begin{tabular}{|c|c|c|c|c|c|c|c|c|c|c|}
\hline & MD (1) & \multicolumn{2}{|c|}{$\mathrm{D}(2)$} & \multicolumn{2}{|r|}{ I (3) } & \multicolumn{2}{|c|}{ A (4) } & \multicolumn{2}{|c|}{ MA (5) } \\
\hline & & $\mathrm{R} 1 \quad \mathrm{R} 2$ & R1 & R2 & R1 & $\mathrm{R} 2$ & R1 & $\mathrm{R} 1$ & R1 & $\mathrm{R} 2$ \\
\hline 1 & $\begin{array}{l}\text { Recoger en una primera parte los } \\
\text { datos personales y la información } \\
\text { relativa a la dieta que se pactó en la } \\
\text { primera visita es útil para disponer } \\
\text { fácilmente de la información inicial. }\end{array}$ & & & & 1 & 0 & 6 & 6 & 6 & 7 \\
\hline 2 & $\begin{array}{l}\text { Resulta útil recoger quien elige la } \\
\text { comida, quien la cocina y donde se } \\
\text { cocina para entender las posibles difi- } \\
\text { cultades que surjan en el seguimiento } \\
\text { de las adaptaciones de la dieta. }\end{array}$ & & & & & & & 2 & 9 & 11 \\
\hline 3 & $\begin{array}{l}\text { Empezar con una pregunta abierta } \\
\text { sobre cómo han ido las comidas y } \\
\text { pedir ejemplos es adecuado para ob- } \\
\text { servar qué información da la unidad } \\
\text { paciente-familia en un primer mo- } \\
\text { mento. }\end{array}$ & & & & & & 3 & & 10 & \\
\hline 4 & $\begin{array}{l}\text { Continuar la recogida de información } \\
\text { a través de una pregunta cerrada de } \\
\text { respuesta dicotómica sobre el segui- } \\
\text { miento de las adaptaciones dietéticas } \\
\text { permite profundizar en este tema, y } \\
\text { valorar la credibilidad que inspira pa- } \\
\text { ciente-familia. }\end{array}$ & & & & & & 8 & & 5 & \\
\hline 5 & $\begin{array}{l}\text { Pedir por el número de veces que el } \\
\text { paciente ha comido alimentos consi- } \\
\text { derados de riesgo es útil para obtener } \\
\text { información sobre el grado de adhe- } \\
\text { rencia a las adaptaciones recomen- } \\
\text { dadas. }\end{array}$ & & & & & & 7 & & 6 & \\
\hline 6 & $\begin{array}{l}\text { En cuanto a los sólidos: considerar } \\
\text { que el calificativo "alguna vez" se } \\
\text { corresponde a } 1-2 \text { veces a la semana } \\
\text { es adecuado. }\end{array}$ & & & & & & 10 & & 3 & \\
\hline 7 & $\begin{array}{l}\text { En cuanto a los sólidos: considerar } \\
\text { que el calificativo "a menudo" se co- } \\
\text { rresponde a 3-7 veces a la semana es } \\
\text { adecuado. }\end{array}$ & & & & 1 & & 8 & & 3 & \\
\hline 8 & $\begin{array}{l}\text { En cuanto a los sólidos: considerar } \\
\text { que el calificativo "siempre" se co- } \\
\text { rresponde a comer alimentos de ries- } \\
\text { go cada día. }\end{array}$ & & & & & & 8 & & 5 & \\
\hline 9 & $\begin{array}{l}\text { Los grupos de alimentos considera- } \\
\text { dos de riesgo y sobre los que se pide } \\
\text { su consumo son adecuados. }\end{array}$ & & 1 & 0 & 1 & 0 & 3 & 5 & 8 & 7 \\
\hline
\end{tabular}




\begin{tabular}{|c|c|c|c|c|c|c|c|}
\hline 10 & $\begin{array}{l}\text { Pedir por la frecuencia con la que el } \\
\text { paciente ha ingerido líquidos en la } \\
\text { viscosidad no recomendada es útil } \\
\text { para obtener el grado de adherencia } \\
\text { a las viscosidades recomendadas en } \\
\text { los líquidos. }\end{array}$ & & & 6 & & 7 & \\
\hline 11 & $\begin{array}{l}\text { En cuanto a los líquidos: considerar } \\
\text { que el calificativo "alguna vez" se } \\
\text { corresponde a } 1-2 \text { veces a la semana } \\
\text { es adecuado. }\end{array}$ & 1 & & 7 & & 5 & \\
\hline 12 & $\begin{array}{l}\text { En cuanto a los líquidos: considerar } \\
\text { que el calificativo "a menudo" se co- } \\
\text { rresponde a 3-7 veces a la semana es } \\
\text { adecuado. }\end{array}$ & 1 & & 8 & & 4 & \\
\hline 13 & $\begin{array}{l}\text { En cuanto a los líquidos: conside- } \\
\text { rar que el calificativo "siempre" se } \\
\text { corresponde a ingerir líquido en la } \\
\text { viscosidad no recomendada cada vez } \\
\text { que bebe. }\end{array}$ & & & 5 & & 7 & \\
\hline 14 & $\begin{array}{l}\text { Recoger la temporalidad en cuanto } \\
\text { a la higiene oral en los ítems nunca, } \\
\text { de forma irregular, } 1-2 \text { veces al día o } \\
\text { después de cada comida es adecuado. }\end{array}$ & & & 8 & 6 & 5 & 7 \\
\hline 15 & $\begin{array}{l}\text { Recoger la forma en que se lleva a } \\
\text { cabo la higiene oral es interesante } \\
\text { para saber si se siguen las indicacio- } \\
\text { nes dadas. }\end{array}$ & & & 9 & & 4 & \\
\hline 16 & $\begin{array}{l}\text { Recoger la forma en que se toma la } \\
\text { medicación es interesante para saber } \\
\text { si se siguen las indicaciones dadas. }\end{array}$ & & & 6 & & 7 & \\
\hline 17 & $\begin{array}{l}\text { Introducir una pregunta para conocer } \\
\text { cómo el paciente vive el hecho de no } \\
\text { poder comer como antes, favorece } \\
\text { la empatía con el paciente y puede } \\
\text { ayudar a dar indicaciones más ade- } \\
\text { cuadas. }\end{array}$ & & 1 & 4 & 2 & 8 & 11 \\
\hline 18 & $\begin{array}{l}\text { La pregunta sobre si el paciente cree } \\
\text { que vale la pena mantener esta dieta } \\
\text { da información sobre la intención de } \\
\text { mantener la dieta recomendada. }\end{array}$ & & 1 & 5 & & 7 & \\
\hline 19 & $\begin{array}{l}\text { La pregunta sobre si el paciente cree } \\
\text { que se siente capaz de mantener esta } \\
\text { dieta da información sobre la autoefi- } \\
\text { cacia de mantener la dieta recomen- } \\
\text { dada. }\end{array}$ & 1 & 2 & 4 & 5 & 6 & 8 \\
\hline 20 & $\begin{array}{l}\text { Recoger el grado de adherencia a las } \\
\text { adaptaciones dietéticas en las cate- } \\
\text { gorías buena, parcial, esporádica, se- } \\
\text { cuencial y no adherencia se considera } \\
\text { adecuado y suficiente. }\end{array}$ & & 2 & 8 & 8 & 2 & 5 \\
\hline
\end{tabular}

$\mathrm{MD}=$ muy desacuerdo $\mathrm{D}=$ desacuerdo $\mathrm{I}=$ indiferente $\mathrm{A}=$ de acuerdo $\mathrm{A}=$ muy acuerdo $\mathrm{R} 1=$ primera ola $\mathrm{R} 2=$ segunda ola 


\section{Metodología de Consenso con panel de expertos}

Una vez finalizada la elaboración del cuestionario de adherencia y previamente a someterlo al análisis de un método de consenso a través de un panel de expertos para obtener una segunda versión acordada, el documento es revisado por una persona externa formada en logopedia y doctora en Didáctica de la Lengua y la Literatura por la Universidad Autónoma de Barcelona. El objetivo de someter el contenido y la forma del cuestionario de adherencia al método Delphi es valorar si el documento realmente permite obtener la información referente al seguimiento o no de las recomendaciones y los motivos en caso de no hacerlo. Este proceso de llegar a un acuerdo entre expertos aporta más calidad y entidad al documento presentado (Pernambuco et al., 2017).

\section{Método Delphi}

El método de consenso utilizado en este estudio es el Delphi, cuyo desarrollo se atribuye a Dalkey y Helmer (Dalkey y Helmer, 1963). Se considera un "método para estructurar un proceso de comunicación grupal que es efectivo para permitir a un grupo de individuos, como un todo, tratar un tema complejo" (Linstone y Turoff, 2002, p. 3). El objetivo es conseguir un consenso de opinión de conocimiento práctico a través de la consulta a un panel de expertos en una determinada área a través de unos cuestionarios (March, García, y Prados, 1998).

El Delphi puede ser especialmente útil en aquellas áreas en las que hay un trabajo previo limitado o incierto (Hardy et al., 2004) en diseño de políticas de toma de decisiones, o en el desarrollo de guías de práctica clínica (Baker, Lovell, y Harris, 2006; Mead y Moseley, 2001). En este caso, la falta de un documento para recoger el seguimiento terapéutico en las adaptaciones de la dieta justifica su razón de ser y de ser consensuado.

El método se desarrolla a través de la administración de unos cuestionarios en diferentes rondas hasta que se logra el consenso. El proceso de feedback posterior a cada ola permite a los expertos reevaluar la primera opinión a partir de la información que proviene del resto de participantes. Habitualmente con tres rondas es suficiente, aunque puede haber más si es necesario para alcanzar el consenso o menos si se llega con más facilidad.

El método Delphi garantiza las siguientes características (Dalkey, 1972; Varela-Ruiz, Díaz-Bravo, y García-Durán, 2012):

a) El anonimato, conseguido a través del número de registro. La comunicación virtual favorece la confidencialidad de las aportaciones de los participantes, proporciona tiempo de reflexión para las respuestas, ayuda a reducir la presión de los individuos dominantes, elimina discusiones directas y permite la inclusión de integrantes de diferentes zonas geográficas.

b) El feedback controlado, el grupo coordinador aporta a los expertos al inicio de cada ronda la opinión global de los ítems del cuestionario a la vez que destaca las posturas discordantes o aquella información significativa para favorecer el diálogo y hacer las aportaciones oportunas (información cuantitativa y cualitativa). 
c) El uso de análisis de técnicas estadísticas, permite reducir la potencial presión de grupo del efecto de la conformidad.

d) Proceso iterativo, la planificación del Delphi en diferentes rondas permite a los expertos aportar su opinión o respuestas a los cuestionarios en cada una de ellas.

\section{Elección del grupo coordinador-administrador}

El grupo coordinador está formado por las personas que establecen el contacto directo con los expertos. Entre sus tareas está el contacto con los expertos a lo largo del proceso, la aportación de los cuestionarios y la gestión del espacio virtual de comunicación. El encargado de hacer la tarea de coordinación es el jefe del área de investigación de la Universidad de Manresa, Universidad de Vic-Universidad Central de Catalunya.

\section{Elección del panel de expertos}

Esta es una tarea muy importante para el éxito del proceso, y por lo tanto es clave hacer una buena elección de los expertos para obtener una buena calidad en los resultados (Delbecq, Van de Ven, y Gustafson, 1975a). Estas personas entendidas se buscan por su liderazgo, por las publicaciones, haciendo los contactos de primera mano (Baker et al., 2006) o por ser miembros del personal profesional (Delbecq, Van de Ven, y Gustafson, 1975b).

Aunque faltan unos criterios claros sobre lo que se considera un experto, en la revisión que Keeney et al. (Keeney, Hasson, y McKenna, 2001) hacen para referirse a experto recogen los conceptos de "individuos informados", "especialistas en un ámbito" o "alguien que tiene un conocimiento sobre un determinado ámbito". De diferentes artículos se recoge que conocimiento, experiencia y habilidad para influir en políticas son los aspectos claves para escoger los expertos (Baker et al., 2006).

Se opta por una muestra bastante homogénea para asegurar la experiencia sobre el tema y la validez de los resultados (Baker et al., 2006), y un total de 13 expertos dado que Delbeck et al. afirman que aunque el número puede ser variable, generalmente entre 10 y 15 en una muestra homogénea es adecuado (Delbecq et al., 1975b).

Según la información obtenida en la revisión bibliográfica se decide buscar una doble representación de expertos. Un grupo de profesionales que ejercen en el ámbito de la logopedia por ser el grupo de profesionales que podrá utilizar el documento en su práctica clínica, y otro representativo del ámbito de la bioética para complementar la aportación desde este punto de vista, clave en cualquier intervención terapéutica. En la tabla 3 se presentan los criterios de selección para ambos grupos. 
Tabla 3. Criterios para la selección del panel de expertos

\begin{tabular}{l|l|l}
\hline Profesión & LOGOPEDIA & BIOETICA \\
\hline Experiencia & $\begin{array}{l}\text { Ser logopedas ejercientes } \\
\text { de } 7 \text { años como mínimo en el ám- } \\
\text { bito de la disfagia }\end{array}$ & $\begin{array}{l}\text { Ser profesionales del ámbito de } \\
\text { la bioética }\end{array}$ \\
\hline Formación & $\begin{array}{l}\text { Demostrar haber hecho formación } \\
\text { lantinua en temas relacionados } \\
\text { con la disfagia }\end{array}$ & $\begin{array}{l}\text { Formar parte de la docencia } \\
\text { universitaria }\end{array}$ \\
\hline $\begin{array}{l}\text { Relación con el do- } \\
\text { cumento }\end{array}$ & $\begin{array}{l}\text { Ser independientes en relación al documento del estudio, no habien- } \\
\text { do participado ni en su elaboración ni en su aplicación }\end{array}$ \\
\hline \hline
\end{tabular}

\section{Presentación del cuestionario}

La investigadora principal contacta vía telefónica con los expertos para obtener su participación en el proyecto. Es el único momento del procedimiento en que tiene contacto directo con los expertos.

En caso de aceptar, el grupo coordinador envía a los expertos una carta personalizada explicativa por correo electrónico con el objeto de estudio, la presentación de la documentación, los procedimientos con los plazos de remisión de las respuestas, y el enlace que les permite acceder al entorno virtual con el CA y la Hoja de instrucciones (Delbecq et al., 1975b).

Paralelamente se pide formalmente por escrito la petición de formar parte del procedimiento y la firma de un documento de Compromiso de Confidencialidad que deben devolver cumplimentado.

Toda la información y los documentos se cuelgan en una página web creada para este motivo.

\section{Temporalización}

La última semana de marzo de 2017 se pide a 15 personas vía correo electrónico la posibilidad de mantener una conversación telefónica en relación a la propuesta de participación en el Método de Consenso, de las que aceptan 13. Se las llama telefónicamente para explicar brevemente el proyecto y su predisposición a colaborar en el proyecto Delphi como expertos. Las 13 personas llamadas confirman la participación y el día 6 de abril de 2017 se les envía la documentación comentada. El plazo para contestar el formulario es hasta el día 10 de mayo de 2017.

Desde el cierre de la primera ola (R1) del Delphi hasta el día 5 de junio se hace un análisis de los comentarios de los expertos. Con fecha de 6 de junio se inicia la segunda ola (R2) y se da de plazo hasta el día 20 de junio. En este procedimiento, se informa a los participantes de los resultados precedentes, tanto aportando los porcentajes de respuestas obtenidos en la R1, como la respuesta de la investigadora a todos los comentarios de los expertos y la nueva propuesta de documento. Se envía nuevamente una carta informando del inicio de la R2, los plazos y los enlaces. 
Para finalizar el estudio se elabora un informe final para los expertos exponiendo los datos globales, el análisis comparativo entre la R1 y la R2, y se adjuntan las respuestas de la investigadora a los comentarios de los expertos en la R2. Asimismo se aporta la última versión del documento consensuado. Toda la información se adjunta en un correo electrónico para cada uno de los expertos el día 6 de septiembre de 2017.

Tabla 4 Temporalización del Método Delphi (año 2017)

\begin{tabular}{|c|c|c|c|c|c|c|c|}
\hline $\begin{array}{l}\text { Última } \\
\text { semana de } \\
\text { marzo }\end{array}$ & $\begin{array}{l}\text { Primera } \\
\text { semana de } \\
\text { abril }\end{array}$ & 6 de abril & $\begin{array}{l}10 \\
\text { de mayo }\end{array}$ & $\begin{array}{l}\text { Hasta } 5 \text { de } \\
\text { junio }\end{array}$ & 6 de junio & 20 de junio & $\begin{array}{l}\text { Del } 20 \text { de } 6 \text { de se- } \\
\text { junio al } 6 \text { de tiembre } \\
\text { setiembre }\end{array}$ \\
\hline $\begin{array}{l}\text { Contacto co- } \\
\text { rreo electró- } \\
\text { nico }\end{array}$ & $\begin{array}{l}\text { Contacto } \\
\text { por } \\
\text { teléfono }\end{array}$ & Inicio R1 & Final R1 & $\begin{array}{l}\text { Análisis de } \\
\text { los resulta- } \\
\text { dos }\end{array}$ & Inicio R2 & Final R2 & $\begin{array}{ll}\text { Análisis de Entrega in- } \\
\text { los resulta- forme final } \\
\text { dos }\end{array}$ \\
\hline
\end{tabular}

\section{Desarrollo práctico y criterios de análisis}

Para cada ronda del cuestionario se registran las respuestas obtenidas en una base de datos securizada Google Drive.

La definición y los criterios de consenso utilizados varían en función de los estudios. En esta propuesta los resultados se analizan a nivel cualitativo y a nivel cuantitativo en función de:

o Media

o Intervalo de confianza

o Content Validity by Item Index (CVI-I) que se recomienda que no sea inferior a 0,78 cuando participan más de seis expertos (Pernambuco et al., 2017; Polit y Tatano Beck, 2006).

Para complementar, la investigadora define como criterio de interpretación de respuesta conflictiva o indicadora de insuficiente consenso la presencia de tres o más expertos que expresen algún grado de desacuerdo.

El análisis a nivel cualitativo se basa en la recogida y respuesta de los comentarios de los expertos.

Siempre que se cumplan los criterios expresados, CVI-I inferior a 0,78, más de tres expertos en desacuerdo, y los comentarios de los expertos lleven a modificar los documentos, se exponen las preguntas o afirmaciones a otra ronda. En caso contrario los ítems no se abren a una próxima ola. Aun así se ha dado respuesta a todos los comentarios expresados por los participantes.

La presentación de los cuestionarios sucesivos se lleva a cabo una vez obtenidos e interpretados los resultados de la anterior ronda. Se presenta a los expertos la siguiente información:

- Porcentaje de cada categoría de respuesta Likert obtenidos para cada afirmación del primer cuestionario.

- Información de los ítems que ya han alcanzado un acuerdo suficiente.

- Todos los comentarios aportados por los expertos.

- Aportaciones de la investigadora principal a los comentarios de los expertos. 


\section{Resultados}

\section{Resultados de los expertos}

Se presenta en la tabla 5 el análisis descriptivo de las características de los expertos. El total de expertos ha sido del género femenino.

El grupo mayoritario de profesionales es del ámbito logopédico o fonoaudiólogo. Siendo los expertos en bioética los docentes, y una de las logopedas que comparte ambas formaciones. También hay una de las profesionales contabilizada como logopeda que de formación inicial es médico. El profesional del ámbito médico tiene la especialización de foniatría. Todos los expertos tienen una licenciatura, una diplomatura o un grado que se distribuye entre las siguientes posibilidades: logopedia, medicina, enfermería, filología y humanidades. Posteriormente con los estudios de postgrado y máster, once de ellos han ido alcanzando las especializaciones en disfagia, motricidad orofacial, neurologopedia, bioética, y nutrición y salud.

Tabla 5 Datos de los expertos

\begin{tabular}{lll}
\hline & $\mathrm{n}$ & $\%$ \\
\hline Edad & & \\
$\quad$ de 29 a 45 años & 6 & 46 \\
$\quad$ de 45 a 61 años & 7 & 54 \\
& & \\
\hline Procedencia & \\
$\quad$ Catalunya & 9 & 70 \\
$\quad$ Portugal & 2 & 15 \\
$\quad$ Sudamérica & 2 & 15 \\
\hline Profesión & & 77 \\
$\quad$ Logopedia & 10 & 8 \\
$\quad$ Medicina & 1 & 15 \\
$\quad$ Docencia & 2 & \\
Nivel de estudios & & 85 \\
$\quad$ Máster y posgrado & 11 & 15 \\
$\quad$ Doctorado & 2 & 15 \\
\hline Años de experiencia profesional & & 39 \\
$\quad$ de 7 a 10 & 2 & 46 \\
$\quad$ de 11 a 20 & 5 & \\
de 21 a 39 & 6 & \\
\hline
\end{tabular}

Los datos muestran que el grupo de expertos cumple los requisitos exigidos, profesionales con una trayectoria mínima de 7 años de experiencia; con formación básica al menos en uno de los dos ámbitos pedidos, el logopédico o el bioético; y con formación continua de especialización en temas relacionados con logopedia y bioética.

\section{Resultados del análisis cuantitativo}

Para favorecer la comprensión de los resultados se utilizan medidas descriptivas paramétricas como la media, el intervalo de confianza y el Content Validity by Item Index (CVI-I). En la tabla 6 se muestran los resultados para las dos olas realizadas. 
Tabla 6. Respuestas de los expertos a las dos olas con medias, intervalos de confianza (IC) y el Content Validity by Item Index (CVI-I)

\begin{tabular}{|c|c|c|c|c|c|c|c|c|}
\hline \multirow[b]{2}{*}{ Ítem* } & \multicolumn{4}{|c|}{ Ola 1} & \multicolumn{4}{|c|}{ Ola 2} \\
\hline & $\mathrm{n}$ & Media & IC95\% & CVI-I & $\mathrm{N}$ & Media & IC95\% & CVI-I \\
\hline 1 & 13 & 4,38 & $(3,99-4,78)$ & 0,92 & 13 & 4,54 & $(4,22-4,85)$ & 1 \\
\hline 2 & 13 & 4,69 & $(4,4-4,98)$ & 1 & 13 & 4,85 & $(4,62-5,07)$ & 1 \\
\hline 3 & 13 & 4,77 & $(4,5-5,03)$ & 1 & & & & \\
\hline 4 & 13 & 4,38 & $(4,08-4,69)$ & 1 & & & & \\
\hline 5 & 13 & 4,46 & $(4,15-4,78)$ & 1 & & & & \\
\hline 6 & 13 & 4,23 & $(3,97-4,5)$ & 1 & & & & \\
\hline 7 & 12 & 4,17 & $(3,8-4,53)$ & 0,92 & & & & \\
\hline 8 & 13 & 4,38 & $(4,08-4,69)$ & 1 & & & & \\
\hline 9 & 13 & 4,38 & $(3,8-4,97)$ & 0,85 & 12 & 4,58 & $(4,26-4,91)$ & 1 \\
\hline 10 & 13 & 4,54 & $(4,22-4,85)$ & 1 & & & & \\
\hline 11 & 13 & 4,23 & $(3,73-4,73)$ & 0,92 & & & & \\
\hline 12 & 13 & 4,15 & $(3,67-4,64)$ & 0,92 & & & & \\
\hline 13 & 12 & 4,58 & $(4,26-4,91)$ & 1 & & & & \\
\hline 14 & 13 & 4,38 & $(4,08-4,69)$ & 1 & 13 & 4,54 & $(4,22-4,85)$ & 1 \\
\hline 15 & 13 & 4,31 & $(4,02-4,6)$ & 1 & & & & \\
\hline 16 & 13 & 4,54 & $(4,22-4,85)$ & 1 & & & & \\
\hline 17 & 13 & 4,54 & $(4,14-4,94)$ & 0,92 & 13 & 4,85 & $(4,62-5,07)$ & 1 \\
\hline 18 & 13 & 4,46 & $(4,14-4,94)$ & 0,92 & & & & \\
\hline 19 & 13 & 4,15 & $(3,56-4,75)$ & 0,77 & 13 & 4,62 & $(4,31-4,92)$ & 1 \\
\hline 20 & 12 & 4 & $(3,62-4,38)$ & 0.83 & 13 & 4.38 & $(4,08-4,69)$ & 1 \\
\hline
\end{tabular}

*El contenido de los ítems puede consultarse en la Tabla 2

En la R1 el CA tiene la siguiente aceptación: 11 de las 20 preguntas tienen un grado de acuerdo del 100\% con un CVI-I de1; 6 de ellas un CVI-I de 0,92; 1 un 0,85 ; 1 un 0,83 ; y en una el CVI-I es 0,77 . De las 20 afirmaciones para valorar el CA sólo la cuestión 19 tiene el CVI-I con un valor indicador de respuesta conflictiva, mostrando los expertos en sus respuestas dos "indiferente" y un "en desacuerdo". En ningún ítem se ha dado la respuesta "muy en desacuerdo". Los ítems 9, 11, 12 y 19 muestran un "en desacuerdo".

En la R1 ha habido un total de 13 cuestiones que además de cumplir los criterios de suficiente consenso, los comentarios de los expertos no han llevado a modificar los documentos (por no aportar soluciones o por falta de consistencia), por lo que no se ha considerado necesario volver a plantearlas en la siguiente ronda. Las 7 afirmaciones restantes piden ser contestadas nuevamente después de haber consultado la nueva propuesta de documentos y argumentaciones de la investigadora. Sigue siendo la escala Likert propuesta en el cuestionario de la R1 y se deja un espacio para nuevos comentarios.

Ante el alto consenso alcanzado en la R1, se decide establecer unos criterios más estrictos y se busca que el porcentaje de "acuerdo" y "muy de acuerdo" no esté por debajo del $90 \%$, que como máximo haya un experto por ítem que pueda mostrar algún grado de desacuerdo y que el CVI-I no sea inferior a 0,92.

Todas las preguntas abiertas en la R2 consiguen un CVI-I de 1. También el grado de "acuerdo" y "muy de acuerdo" ha sido del 100\%, y no ha habido ningún experto que haya discrepado. Sólo se observa una observación terminológica de la afirmación 20 que recoge las diferentes categorías para describir el grado de adherencia en la que un experto manifiesta la poca claridad del término "secuencial" cuando se definen los grados de adherencia. 


\section{Análisis cualitativo de los resultados-Cambios en los documentos}

Las observaciones de los expertos se recogen tanto del apartado de comentarios abiertos de cada pregunta como de la pregunta final. Su contenido es sobre cuestiones de organización y titulación de la primera parte del documento, de aspectos formales de alguna de las preguntas, del modo de pasar el cuestionario (evitando pasar las preguntas de seguimiento con según qué alimentos si previamente no se había indicado su recomendación), de los calificativos para recoger la frecuencia de ingesta, y en relación a las opciones finales para valorar el grado de adherencia.

Los comentarios de los expertos ayudan a mejorar por una parte la forma y el contenido del documento; por otra parte, para definir con más concreción la forma de utilizarlo, se añaden estas observaciones en la Hoja de instrucciones. En la tabla 7 se especifican los cambios introducidos en el documento a partir de los comentarios de los expertos.

Tabla 7. Modificaciones en el documento de adherencia

\begin{tabular}{|c|c|}
\hline ○ & Se añade el calificativo "médico" al "diagnóstico". \\
\hline o & Se cambia "fecha de orientación" por "fecha de entrevista de adherencia". \\
\hline ○ & $\begin{array}{l}\text { Se pone una parrilla para facilitar la distribución de la información inicial y se añade el } \\
\text { título "Indicaciones dadas". }\end{array}$ \\
\hline ○ & Se modifica el concepto de "dieta logopeda" por "dieta adaptada". \\
\hline o & $\begin{array}{l}\text { En el apartado de los "líquidos" de las "Indicaciones dadas" se añade el concepto de "ge- } \\
\text { latina". }\end{array}$ \\
\hline o & $\begin{array}{l}\text { En el apartado de "Medicación" de las "Indicaciones dadas" se sustituye "aplastada" por } \\
\text { "triturada" y en las "fórmulas líquidas" se especifica el grado de viscosidad (néctar, miel, } \\
\text { pudín). }\end{array}$ \\
\hline o & $\begin{array}{l}\text { En el apartado de "Higiene oral" de las "Indicaciones dadas", se añade el "colutorio" y un } \\
\text { apartado de "otros". }\end{array}$ \\
\hline ○ & $\begin{array}{l}\text { En la opción de "otros" del "Lugar habitual de comidas" se añade una línea punteada para } \\
\text { facilitar la escritura. }\end{array}$ \\
\hline o & Se mejora la redacción en castellano. \\
\hline ○ & $\begin{array}{l}\text { Se introduce una pregunta entre la } 11 \text { y la } 13 \text { referente a la ingesta de alimentos sólidos en } \\
\text { positivo Desde que le dieron el alta del hospital, ¿con qué frecuencia ha tomado los alimen- } \\
\text { tos recomendados (pastoso, blando, homogéneo)? “. Pasan de } 17 \text { a } 18 \text { ítems. }\end{array}$ \\
\hline ○ & $\begin{array}{l}\text { En la "Higiene oral" se sustituye la medida de "después de cada comida" por "después de } \\
\text { cada comida principal". }\end{array}$ \\
\hline ○ & $\begin{array}{l}\text { La pregunta } 15 \text { “Cómo vive el hecho de no poder comer como antes?” que ha pasado a ser } \\
\text { la } 16 \text {, se reformula en positivo ¿Cómo vive los cambios introducidos en su dieta?". }\end{array}$ \\
\hline ○ & $\begin{array}{l}\text { En el apartado de definición del grado de adherencia se añade "no recomendados" después } \\
\text { de "sólidos y líquidos" en los grados de adherencia siguientes: "buena", "esporádica" y "no } \\
\text { adherencia". }\end{array}$ \\
\hline o & $\begin{array}{l}\text { La observación de la pregunta } 9 \text { sobre el hecho de que no todos los grupos de alimentos } \\
\text { son considerados de riesgo para todos los pacientes se responde introduciendo en la Hoja } \\
\text { de instrucciones la posibilidad de preguntar por el grupo de alimentos de riesgo para cada } \\
\text { paciente específicamente. }\end{array}$ \\
\hline
\end{tabular}


Otras observaciones de los expertos son contestadas sin que tengan una repercusión de modificación en el instrumento propuesto. Se informa a los expertos acerca de las observaciones de las preguntas 17 y 18 sobre la pregunta de la vivencia de los cambios de dieta y sobre la intención de mantener la dieta respectivamente, de que ambas están resueltas en las indicaciones redactadas en la Hoja de instrucciones. También cuando se cuestiona la expresión "sentirse capaz" incluida en la pregunta 19, la investigadora argumenta que según la Teoría Social Cognitiva de Bandura el concepto es clave en el mantenimiento de las conductas esperadas a adherir.

\section{Discusión}

La propuesta del Cuestionario de Adherencia (CA) se apoya en la inexistencia de cuestionarios para recoger la adherencia en pacientes con disfagia que precisan de adaptaciones dietéticas. Aunque existen tests de adherencia para recoger el seguimiento en cuanto a medicaciones (Haynes et al., 1980; Herrera Carranza, 2001) o también el seguimiento para otro tipo de dietas (Bentley et al., 2009; Centro de Investigación Biomédica en Red, 2015; Russell et al., 2017), no es así para el dominio de la adherencia de las personas con disfagia a las adaptaciones dietéticas.

El CA obtiene una buena verificación de la evidencia de validez basada en el contenido gracias al amplio consenso de los expertos en cuanto a que el instrumento recoge la adherencia de las personas con disfagia a las adaptaciones dietéticas. El procedimiento Delphi para evaluar el CA y llegar a un consenso para ajustar los aspectos semánticos, sintácticos y contextuales de la versión inicial del CA ha requerido dos rondas. El espacio creado para intercambiar las opiniones entre todos los expertos y la investigadora principal ha revertido de forma positiva para mejorarlo en cuanto a su redacción, representatividad y relevancia. Las opiniones de los expertos invitados se han analizado tanto cuantitativamente como cualitativamente.

Se cumple con los requisitos exigidos para hacer una buena elección de los expertos. Tanto en cuanto al número de expertos que ha sido de trece, dato comprendido entre diez y quince que es lo recomendado (Delbecq et al., 1975b), como por sus características, años de experiencia superior a 7 años, formación en logopedia o bioética y haber realizado formación continua.

La exposición de las estrategias para la valoración de la validez en cuanto al contenido de la creación de instrumentos en fonoaudiología incluye también, a parte de la revisión de la literatura, la experiencia empírica de los investigadores y las entrevistas con informantes clave como los especialistas, las entrevistas con la población objetivo utilizando grupos focales (Pernambuco et al., 2017). Si bien se han llevado a cabo el primer grupo de estrategias, no ha sido así con la de utilizar entrevistas con la población objetivo, ya que en esta primera etapa se quería conocer la opinión exclusivamente de los expertos.

A pesar del número elevado de preguntas con CVI-I superior a 0,78 , que representa el valor por debajo del cual las respuestas de un grupo superior a seis expertos se consideraría no adecuado, la mayoría de las observaciones contenidas en las respuestas de los expertos ha desencadenado una revisión de la primera versión del CA para poder mejorarlo. Existen cuatro ítems de la R1 en los que un experto ha discrepado, pero no han aportado alternativas. Los comentarios han sido argumentados por la investigadora en la R1 sin llevar a modificar el documento propuesto, razón 
por la cual no se han abierto a la R2. Dos de ellos el 11 y el 12 han sido puntuados con "en desacuerdo". Su contenido versa sobre los términos utilizados para definir la frecuencia de ingesta de los líquidos. Los ítems 7 y 18 valorados con "indiferente" se refieren, el primero al término "a menudo" utilizado para definir la frecuencia de ingesta de los sólidos, y el segundo a la pregunta sobre si vale la pena mantener la dieta recomendada.

Siguiendo los criterios iniciales de respuestas conflictivas, en la R1 sólo el ítem 19 se define como conflictivo con un ICV-I de 0,77. La pregunta sobre si el paciente cree que se siente capaz de mantener la dieta proporciona información sobre la autoeficacia de mantener la dieta recomendada, aspecto clave en el mantenimiento del cumplimiento terapéutico (Bandura, 1987). Por este motivo la pregunta no se modifica y con la argumentación proporcionada por la investigadora, en la R2 se consigue un CVI-I de 1.

Todas las preguntas que se abren para la $\mathrm{R} 2$ reciben una puntuación del CVI-I de 1. Sólo aparece una observación terminológica en la afirmación 20 que recoge las diferentes categorías para describir el grado de adherencia, en la que el experto manifiesta la poca claridad del término "secuencial" cuando se definen los grados de adherencia. Se argumenta que las categorías son extraídas de la propuesta de Vilaplana y colaboradores (Vilaplana et al., 2012), y que el término define con precisión la situación de iniciar la adherencia manteniéndose en las indicaciones y abandonarlas posteriormente, por lo que se ha considerado más oportuno no modificarlas.

\section{Limitaciones de la propuesta}

Se reconoce la necesidad de llevar a cabo una prueba piloto con pacientes para obtener un mayor grado de validez de contenido.

Se observa una limitación, y no tanto por el documento en sí, sino por la presión asistencial y la falta de recursos actuales, del escaso tiempo disponible para desarrollar la tarea profesional con la calidad que sería deseable. El objetivo de centrarse en la persona, y favorecer el diálogo en el encuentro terapéutico exige tiempo, recurso no siempre disponible en la práctica asistencial habitual.

Se destaca la necesidad de tener la oportunidad de hacer el seguimiento terapéutico a los pacientes a los que se orienta en la dieta. En ocasiones, el seguimiento terapéutico no puede asegurarse y queda la indicación en relación con la dieta como una intervención aislada.

\section{Conclusiones}

En primer lugar, se considera que la creación de un Cuestionario de Adherencia para valorar el seguimiento terapéutico en pacientes con disfagia que precisan adaptaciones dietéticas es una aportación en el manejo de estos pacientes, ya que en la búsqueda previa no se han encontrado instrumentos similares al presentado. Por este motivo se concluye que la propuesta de crear un documento para hacer el seguimiento terapéutico en pacientes que presentan una alteración de la deglución que no les impide alimentarse por vía oral es nueva y útil en el campo clínico. 
En segundo lugar, la iniciativa de someter la propuesta inicial del Cuestionario de Adherencia al método de consenso del Delphi da entidad y robustez al instrumento. El hecho de haber sido examinado por un grupo de expertos en la atención a la disfagia y también del campo de la bioética ha permitido mejorar la propuesta inicial y dotarlo de más adecuación y conformidad, convirtiéndose en una herramienta más satisfactoria.

\section{Agradecimientos}

El trabajo se desarrolla en el contexto de la Tesis doctoral "Bioètica en la presa de decisions: Adaptacions dietètiques en la disfàgia" dirigida per Dra. Margarita Boladeras y Dra. Josefina Goberna. Agradezco al Dr. Albert Espelt de la UVic-UCC la revisión metodológica del manuscrito.

\section{Referencias bibliográficas}

Aguilar Salmeron R. (s. f.). Administración de medicamentos en personas con dificultades para tragar. Centro de información de medicamentos de Cataluña. Recuperado 2 de noviembre de 2017, a partir de http://www.cedimcat.info/html/es/dir2455/doc26581.html

Alexandre, N. M. C., y Coluci, M. Z. O. (2011). Validade de conteúdo nos processos de construção e adaptação de instrumentos de medidas. Ciência \& Saúde Coletiva, 16(7), 3061-3068. https://doi.org/10.1590/S1413-81232011000800006

Almirall, J., Cabré, M., y Clavé, P. (2007). Aspiration pneumonia. Medicina Clínica, 129(11), 424-432.

American Educational Research Association, American Psychological Association, y National Council on Measurement in Education. (2014). Standards for educational and psychological testing. Washington.

Baker, J., Lovell, K., y Harris, N. (2006). How expert are the experts? An exploration of the concept of «expert» within Delphi panel techniques. Nurse researcher, 14(1), 59-70. https://doi.org/10.7748/nr2006.10.14.1.59.c6010

Bandura, A. (1987). Pensamiento y acción: Fundamentos sociales. Barcelona: Martínez Roca.

Bentley, B., Lennie, T. A., Biddle, M., Chung, M. L., y Moser, D. K. (2009). Demonstration of psychometric soundness of the Dietary Sodium Restriction Questionnaire in patients with heart failure. Heart \& lung : the journal of critical care, 38(2), 121-128. https://doi. org/10.1016/j.hrtlng.2008.05.006

Bleeckx, D. (2004). Disfagia: Evaluación y reeducación de los trastornos de la deglución. Madrid: Mc Graw Hill Interamericana.

Centro de Investigación Biomédica en Red. (2015). Estudio Predimed: cumplimiento de la dieta. Recuperado 2 de septiembre de 2017, a partir de http://www.predimed.es/investigators-tools.html

Clavé, P., y Cichero, J. A. Y. (2012). Stepping stones to living well with dysphagia. Barcelona: Nestlé Nutrition Institute.

Clavé, P., y García Peris, P. (2011). Guía de diagnóstico y de tratamiento nutricional y rehabilitador de la disfagia orofaríngea. Barcelona: Editorial Glosa. 
Clavé, P., Verdaguer, A., y Arreola, V. (2005). Disfagia orofaríngea en el anciano. Medicina Clinica, 124(19), 742-748. https://doi.org/10.1016/j.medcli.2008.12.029

Dalkey, N. C. (1972). The Delphi method: An experimental study of group opinion. En Dalkey y \& D. S. D. L. Rourke, R. Lewis (Eds.), Studies in the quality of life: Delphi and decision-making (pp. 13-54). Lexington, MA: Lexington Books.

Dalkey, N. C., y Helmer, O. (1963). An experimental application of the Delphi method to the use of experts. Management science, 9(3), 458-467. https://doi.org/10.1287/mnsc.9.3.458

Delbecq, A. L., Van de Ven, A. H., y Gustafson, D. H. (1975a). Group decision making in modern organizations. En Group Techniques for Program Planning (pp. 1-14). Glenview, Illinois: Scott, Foresman, and Co.

Delbecq, A. L., Van de Ven, A. H., y Gustafson, D. H. (1975b). The Delphi techniques. En Group techniques for program planning (pp. 83-107). Glenview, Illinois: Scott, Foresman, and Co.

Elliot, J. (2000). La investigación-acción en educación (4. ${ }^{a}$ ed.). Madrid: Ediciones Morata.

Fishbein, M., y Ajzen, I. (1975). Belief, attitude, intention, and behavior: An introduction to theory and research. Reading, MA: Addison-Wesley.

Hardy, D. J., O’Brien, A. P., Gaskin, C. J., O’Brien, A. J., Morrison-Ngatai, E., Skews, G., ... McNulty, N. (2004). Practical application of the Delphi technique in a bicultural mental health nursing study in New Zealand. Journal of advanced nursing, 46(1), 95-109. https://doi.org/10.1111/j.1365-2648.2003.02969.x

Haynes, R. B., Taylor, D. W., Sackett, D. L., Gibson, E. S., Bernholz, C. D., y Mukherjee, J. (1980). Can simple clinical measurements detect patient noncompliance? Hypertension, 2(6).

Hernández Martín, J., Correa Ballester, M., Vial Escolano, R., Forcano García, M., Gómez Navarro, R., y González García, P. (2013). Adaptación de la guía farmacoterapéutica de un hospital sociosanitario a pacientes con disfagia. Farmacia hospitalaria, 37(3), 198208. https://doi.org/10.7399/FH.2013.37.3.577

Herrera Carranza, J. (2001). El incumplimiento terapéutico como problema relacionado con medicamentos diferenciado. Pharmaceutical Care España, 3(6), 446-448.

Ickenstein, G. W. (2011). Diagnosis and treatment of neurogenic dysphagia. Bremen: UNIMED Science.

Jabary, N. S., Castrodeza, J., Monfa, J. M., y Sousa, F. (1999). Validación de un nuevo test para determinar el cumplimiento terapéutico en pacientes hipertensos: Test Hermes. $\mathrm{Hi}$ pertensión, 16, 298-303.

Janz, N. K., y Becker, M. H. (1984). The health belief model: A decade later. Health education quarterly, 11(1), 1-47.

Keeney, S., Hasson, F., y McKenna, H. P. (2001). A critical review of the Delphi technique as a research methodology for nursing. International Journal of Nursing Studies, 38(2), 195-200. https://doi.org/10.1016/S0020-7489(00)00044-4

Linstone, H. A., y Turoff, M. (2002). The Delphi method: Techniques and applications. Reading: Addison-We.

March, J. C., García, M. M., y Prados, A. (1998). Los métodos de investigación y evaluación en la promoción de la salud. En F. Martínez, P. Antó, P. Castellanos, M. Gili, P. Marset, y V. Navarro (Eds.), Salud pública (pp. 363-384). Madrid: McGraw- Hill.

Mead, D., y Moseley, L. (2001). The use of the Delphi as a research approach. Nurse researcher, 8(4), 4-23. https://doi.org/10.7748/nr2001.07.8.4.4.c6162

Morisky, D. E., Green, L. W., y Levine, D. M. (1986). Concurrent and predictive validity of a self-reported measure of medication adherence. Medical care, 24(1), 67-74. 
Ortega, O., Cabré, M., y Clavé, P. (2014). Oropharyngeal dysphagia: Aetiology and effects of ageing. Journal of Gastroenterology and Hepatology Research, 3(5), 1049-1054.

Pernambuco, L., Espelt, A., Magalhães Junior, H. V., y Lima, K. C. de. (2017). Recomendações para elaboração, tradução, adaptação transcultural e processo de validação de testes em Fonoaudiologia. Communication Disorders, Audiology and Swallowing, 29(3), 2-5. https://doi.org/10.1590/2317-1782/20172016217

Polit, D. F., y Tatano Beck, C. (2006). The Content Validity Index: Are you sure you know what's being reported? Critique and recommendations. Research in nursing \& health, 29, 489-497.

Prados, A., y Piera, G. (2014). Fitxes de recomanacions dietètiques en les alteracions digestives i la disfàgia de la BBS. Circular farmacèutica, 72(3), 27-31.

Prochaska, J. O. (1979). Systems of psychotherapy: A transtheoretical analysis. Illinois: Dorsey Press.

Rosenstock, I. M. (1974). Historical origins of the health belief model. Health education monographs, 2(4), 328-335.

Russell, J., Flood, V., Sadeghpour, A., Gopinath, B., y Mitchell, P. (2017). Total Diet Score as a valid method of measuring diet quality among older adults. Asia Pacific Journal of Clinical Nutrition, 26(2), 212-219.

Scottish Intercollegiate Guidelines Network (Ed.). (2004). Management of patients with stroke: Identification and management of dysphagia. Edimburgh: Scottish Intercollegiate Guidelines Network.

Sireci, S. G. (1998). The construct of content validity. Social Indicators Research, 45, 83117. https://doi.org/10.1023/A:1006985528729

Smith Hammond, C. A., y Goldstein, L. B. (2006). Cough and aspiration of food and liquids due to oral-pharyngeal dysphagia. Chest, 129(1 Suppl), 154S-168S. https://doi. org/10.1378/chest.129.1

Stenhouse, L. (1996). La investigación como base de la enseñanza (3. ${ }^{\text {a }}$ ed.). Madrid: Ediciones Morata.

Tójar Hurtado, J. C. (2006). Investigación cualitativa: Comprender y actuar. Madrid: Editorial La Muralla.

Varela-Ruiz, M., Díaz-Bravo, L., y García-Durán, R. (2012). Descripción y usos del método Delphi en investigaciones del área de la salud. Revista de investigación en educación médica, 1(2), 90-95. Recuperado a partir de http://riem.facmed.unam.mx/node/204

Vilaplana, C., González, F., y Ordoñana, J. R. (2012). Adherencia al tratamiento: Una revisión desde la perspectiva farmacéutica. Pharmaceutical Care España, 14(6), 249-55. Recuperado a partir de http://pharmcareesp.com/index.php/PharmaCARE/article/view/97

Wright, D., Chapman, N., Foundling-Miah, M., Greenwall, R., Griffith, R., Guyon, A., y Merriman, H. (2015). Consensus guideline on the medication management of adults with swallowing difficulties. Chesham: MGP: Ltd. Recuperado a partir de https://www. rosemontpharma.com/sites/default/files/Adult dysphagia full guideline \%5B2015 update $\% 5$ D.pdf 


\section{CUESTIONARIO DE ADHERENCIAA LAS ADAPTACIONES DIETÉTICAS EN DISFAGIA}

Nombre y apellidos:

Diagnóstico médico:

Fecha entrevista de adherencia:

\begin{tabular}{|c|c|c|c|c|}
\hline \multicolumn{4}{|c|}{ INDICACIONES DADAS } & e la orientación: \\
\hline \multicolumn{2}{|l|}{ Dieta pastosa } & \multicolumn{3}{|c|}{ Dieta adaptada } \\
\hline \multirow{6}{*}{$\begin{array}{l}\text { Líquidos: } \\
\text { Agua con gas } \\
\text { Néctar } \\
\text { Miel } \\
\text { Pudin } \\
\text { Gelatinas }\end{array}$} & \multicolumn{3}{|c|}{ Medicación: } & Higiene oral: \\
\hline & \multicolumn{2}{|l|}{ Entera } & Triturada & $\begin{array}{l}\text { Cepillo- pasta de dientes y/o } \\
\text { colutorio enjuagando }\end{array}$ \\
\hline & \multicolumn{3}{|c|}{ Mezclada con puré } & $\begin{array}{l}\text { Con la cabeza inclinada hacia } \\
\text { abajo enjuagando }\end{array}$ \\
\hline & \multicolumn{3}{|c|}{ Mezclada con líquidos } & Cepillo húmedo sin enjuagar \\
\hline & \multicolumn{3}{|c|}{ Fórmulas líquidas espesadas: } & Con una gasa húmeda escurrida \\
\hline & \multicolumn{3}{|c|}{ Néctar Miel Pudin } & Otras: \\
\hline
\end{tabular}

¿Quién escoge los alimentos? ¿Quién decide las comidas?

¿Quién prepara la comida?

\begin{tabular}{|c|c|c|c|c|c|}
\hline \multicolumn{6}{|c|}{$\begin{array}{l}\text { Lugar habitual de las comidas: } \\
\text { amigos } \quad \text { restaurante }\end{array}$} \\
\hline $\begin{array}{l}\text { Etapa de cambio: } \\
\text { acción }\end{array}$ & \multicolumn{3}{|c|}{$\begin{aligned} \text { precontemplación } & \text { contemplación } \\
\text { mantenimiento } & \text { finalización }\end{aligned}$} & \multicolumn{2}{|c|}{ preparación al cambio } \\
\hline 1 & \multicolumn{3}{|c|}{$\begin{array}{l}\text { ¿Cómo han ido las comidas desde que se le dio el alta del hospital? } \\
\text { Explique las comidas de un día }\end{array}$} & & \\
\hline \multirow[t]{2}{*}{2} & \multirow{2}{*}{\multicolumn{3}{|c|}{$\begin{array}{l}\text { Desde que le dieron el alta del hospital, ¿ha seguido las indicaciones } \\
\text { recomendadas? }\end{array}$}} & Sí & No \\
\hline & & & & \multicolumn{2}{|l|}{ ¿Por qué? } \\
\hline \multicolumn{2}{|c|}{$\begin{array}{l}\text { Desde que le dieron el alta del hospital, } \\
\text { ¿cuántas veces ha comido: }\end{array}$} & Nunca & $\begin{array}{l}\text { Alguna vez } \\
(1-2 \text { semana) }\end{array}$ & $\begin{array}{l}\text { A menudo } \\
\text { (entre 3-7 sem.) }\end{array}$ & Siempre \\
\hline 3 & $\begin{array}{l}\text { alimentos fibrosos como por ejem- } \\
\text { plo alcachofas? }\end{array}$ & & & & \\
\hline 4 & $\begin{array}{l}\text { alimentos duros como carne con } \\
\text { hueso por ejemplo cordero? }\end{array}$ & & & & \\
\hline 5 & $\begin{array}{l}\text { alimentos con pieles como por } \\
\text { ejemplo uva? }\end{array}$ & & & & \\
\hline 6 & $\begin{array}{l}\text { alimentos de dobles consistencias } \\
\text { como por ejemplo sopas? }\end{array}$ & & & & \\
\hline 7 & $\begin{array}{l}\text { alimentos granulados como por } \\
\text { ejemplo arroz? }\end{array}$ & & & & \\
\hline 8 & $\begin{array}{l}\text { alimentos que desprenden líquido } \\
\text { como por ejemplo naranjas? }\end{array}$ & & & & \\
\hline
\end{tabular}




\begin{tabular}{|c|c|c|c|c|c|c|}
\hline 9 & $\begin{array}{l}\text { alimentos secos como por ejemplo } \\
\text { pechuga de pollo a la plancha? }\end{array}$ & & & & & \\
\hline 10 & $\begin{array}{l}\text { alimentos que se esparcen como } \\
\text { por ejemplo pan tostado? }\end{array}$ & & & & & \\
\hline 11 & $\begin{array}{l}\text { alimentos pegajosos como por } \\
\text { ejemplo madalenas? }\end{array}$ & & & & & \\
\hline 12 & $\begin{array}{l}\text { Desde que le dieron el alta del hos- } \\
\text { pital, ¿con qué frecuencia ha to- } \\
\text { mado los alimentos recomendados } \\
\text { (pastoso, blando, homogéneo)? }\end{array}$ & Nun & & $\begin{array}{l}\text { Alguna vez } \\
\text { (habitualmente no, } \\
\text { pero alguna vez sí) }\end{array}$ & \begin{tabular}{|l|} 
A menudo \\
(habitualmen- \\
te, pero alguna \\
vez no)
\end{tabular} & Siempre \\
\hline 13 & $\begin{array}{l}\text { Desde que le dieron el alta del } \\
\text { hospital, icon qué frecuencia ha } \\
\text { tomado los líquidos en la viscosi- } \\
\text { dad recomendada? }\end{array}$ & Nun & & $\begin{array}{l}\text { Alguna vez } \\
\text { (1-2 semana) }\end{array}$ & \begin{tabular}{|l|} 
A menudo \\
(entre \\
sem.)
\end{tabular} & Siempre \\
\hline \multirow[t]{2}{*}{14} & Se lava los dientes & \multicolumn{2}{|c|}{ Nunca } & De forma irregular & $\begin{array}{lll}1-2 & \text { veces al } \\
\text { día } & & \end{array}$ & $\begin{array}{l}\text { Después de } \\
\text { cada comida } \\
\text { principal }\end{array}$ \\
\hline & \multicolumn{6}{|l|}{ ¿Cómo? } \\
\hline \multirow{2}{*}{15} & ¿Cómo se toma la medicación? & \multicolumn{2}{|c|}{ Entera } & \multicolumn{3}{|l|}{ Triturada con puré } \\
\hline & \multicolumn{6}{|l|}{ Fórmulas líquidas espesadas } \\
\hline 16 & $\begin{array}{l}\text { ¿Cómo vive los cambios } \\
\text { introducidos en su dieta? }\end{array}$ & & & & & \\
\hline 17 & $\begin{array}{l}\text { ¿Cree que vale la pena mantener } \\
\text { esta dieta? }\end{array}$ & Sí & No & ¿Por qué? & & \\
\hline 18 & $\begin{array}{l}\text { ¿Cree que se siente capaz de } \\
\text { mantener esta dieta? }\end{array}$ & Sí & No & ¿Por qué? & & \\
\hline
\end{tabular}

\section{Grado de adherencia:}

Buena: cumplimiento total (sólidos y líquidos no recomendados: nunca; se lava los dientes: después de cada comida).

Parcial: sólo una parte (respuestas variadas de la 3 a la 13; se lava los dientes: nunca, de forma irregular, 1-2 veces al día).

Esporádica: frecuencia variada (sólidos y líquidos no recomendados: alguna vez, a menudo; se lava los dientes: de forma irregular).

Secuencial (durante un tiempo sí, y a partir de un momento deja de seguir las recomendaciones).

No adherencia: incumplimiento total (sólidos y líquidos no recomendados: siempre; se lava los dientes: nunca).

(Se valora la adherencia en sólidos, líquidos y en la administración de la medicación, en función de la prescripción inicial) 


\section{HOJA DE INSTRUCCIONES \\ Cuestionario de adherencia}

El cuestionario de adherencia, que tiene el objetivo de recoger el grado de adherencia a las adaptaciones dietéticas pactadas, se define en cinco secciones:

- Recogida de datos: para obtener información sobre los datos básicos del paciente en relación con la patología, y la alimentación (dieta pactada, persona que elige los alimentos y que cocina, lugares donde se come, etapa de cambio).

- Recogida del seguimiento terapéutico, con la frecuencia de ingestión de alimentos o líquidos no recomendados, y también de los recomendados. También de cómo se administra la medicación, y cómo se hace la higiene oral.

- Obtención de la opinión de la vivencia del paciente con el cambio de dieta.

- Obtención de la opinión del paciente y de la familia sobre la declaración de intenciones y la autoeficacia en mantener la dieta pactada.

- Definición del grado de adherencia (buena, parcial, esporádica, secuencial, no adherencia).

Existen unos aspectos de carácter general del cuestionario para recoger la adherencia que se deben tener presentes. En primer lugar, el test se basa en la honestidad de paciente-familia al responder, ya que las preguntas van dirigidas a ellos. En segundo lugar, con respecto a la administración del cuestionario, aunque éste puede ser auto administrado, el interés principal es recoger las respuestas a través de la entrevista personal, de modo que sirva de asistencia al entrevistador. En tercer lugar, incidir en que el interés es recoger de forma cualitativa la información sobre el seguimiento o no de las adaptaciones en la dieta y los motivos. En ningún caso el ánimo es obtener una puntuación numérica. En cuarto lugar, el cuestionario pretende obtener la adherencia tanto de personas a las que se les ha indicado una dieta totalmente triturada o pastosa, como las que pueden alimentarse con alimentos sólidos adaptados. En quinto y último lugar, a pesar del deterioro cognitivo que pueda tener el paciente, siempre se tiene en cuenta su opinión ya que habitualmente el paciente sabe lo que le gusta o quiere comer, y así lo muestra a menudo durante las comidas.

Las preguntas van dirigidas al paciente si cognitivamente está bien, y también al familiar, ya que puede existir falta de concordancia entre sus respuestas. Las respuestas se recogen en el cuestionario de la siguiente forma: rodeando la respuesta adecuada cuando haya opciones, redactando en caso de preguntas abiertas y añadiendo el alimento en cuestión en la columna de la frecuencia correspondiente en las preguntas de la 3 a la 11. Las respuestas del paciente se recogen en color rojo y las respuestas del familiar en color verde.

En el caso de las preguntas sobre la adherencia a los alimentos considerados de riesgo, preguntas de la 3 a la 11, pueden obviarse aquellas que no corresponden a una orientación dada previamente para un paciente en concreto. Por ejemplo si a un paciente solo se le recomienda no tomar dobles consistencias, se le puede formular la pregunta 6 , pero no el resto. 